Chronic Obstructive Pulmonary Diseases: Journal of the COPD Foundation

\author{
Original Research
}

\title{
Identifying Patients With COPD at High Risk of Readmission
}

\author{
Jacob E. Simmering, $\mathrm{MS}^{1}$ Linnea A. Polgreen, $\mathrm{PhD}^{1}$ Alejandro P. Comellas, $\mathrm{MD}^{2}$ Joseph E. Cavanaugh, $\mathrm{PhD}^{3}$ \\ Philip M. Polgreen, MD, MPH ${ }^{2}$
}

\begin{abstract}
Background: Readmission within 30 days of a COPD hospitalization is a common measure of performance for COPD care. However, most studies of COPD readmission risk have been constrained to a single data source, private payer claims, or Medicare claims data, making it difficult to generalize results from these studies to other populations. The purpose of this study was to examine the risk for readmission within 30 days from time of discharge in patients with COPD using the Healthcare Cost and Utilization Project (HCUP) State Inpatient Database for California for the years 2005-2011. This statewide dataset allows us to consider all readmissions for COPD regardless of age or payer status.
\end{abstract}

Methods: The total dataset included 28,265,070 visits among 17,918,374 patients over 480 hospitals. We identified patients with a hospitalization, a primary diagnosis related to COPD, age 40 or older, and discharged alive. We found 286,313 hospitalizations that matched this definition and included information on covariates such as comorbidities, age, and insurance status. To characterize the joint associations of these covariates with readmission within 30 days, we used a generalized linear model.

Results: Patients aged 40-64 are more likely to be readmitted to the hospital within 30 days of a COPD-related hospitalization than patients 65 and older. This effect persists after adjustment for patient severity, comorbidities, payer, and demographics.

Our model featured an interaction of age with insurance type. We found that younger patients (aged 40-64) on public insurance have the highest readmission rates: $14.77 \%$ for Medicare and $16.27 \%$ for Medicaid. However, younger patients with private insurance have the lowest readmission rates at $8.25 \%$. Additional significant covariates included whether or not the patient left against medical advice, and diagnoses of congestive heart failure and diabetes.

In addition, we found that although admissions for COPD were highest in the winter, this is not true for COPD readmissions, which peak in summer. Also, inpatient mortality for patients admitted for COPD decreased from approximately $3 \%$ to $1.25 \%$ over the study period.

Conclusion: Our results demonstrate that many of the risk factors for readmission may be dependent on the data source used. Furthermore, many of the strongest predictors are clearly related to the patients themselves. This observation may help explain why prior programs to reduce readmissions have had limited success.

\footnotetext{
Abbreviations: chronic obstructive pulmonary disease, COPD; acute COPD exacerbations, AECOPD; Agency for Healthcare Research and Quality, AHRQ; Healthcare Cost and Utilization Project, HCUP; generalized linear model, GLM; odds ratio, OR; confidence interval, CI Funding Support: This work was supported by the National Heart, Lung, and Blood Institute at the National Institutes of Health [grant number K25HL 122305 to LAP] and the University of Iowa eHealth and eNovation Center. The sponsors had no role in this manuscript. Date of Acceptance: May 18, 2016

Citation: Simmering JE, Polgreen LA, Comellas AP, Cavanaugh JE, Polgreen PM. Identifying patients with COPD at high risk of readmission. Chronic Obstr Pulm Dis (Miami). 2016;3(4):729-738. doi: http://dx.doi.org/10.15326/jcopdf.3.4.2016.0136
} 

1 University of Iowa, Department of Pharmacy Practice and Science,
Iowa City

2 University of Iowa, Department of Internal Medicine, Iowa City

3 University of Iowa, Department of Biostatistics, Iowa City

\section{Address correspondence to:}

Philip M. Polgreen, MD, MPH

Email: philip-polgreen@uiowa.edu

Phone: 319-356-1616

\section{Keywords:}

COPD, readmission, inpatient, adult, patient characteristics

\section{Introduction}

Chronic obstructive pulmonary disease (COPD) is a major cause of morbidity and mortality. ${ }^{1,2}$ Patients with COPD have different clinical, imaging, and biological phenotypes. ${ }^{3-6}$ One of these phenotypes is acute COPD exacerbations (AECOPD), ${ }^{7,8}$ and admissions for exacerbations account for the majority of costs associated with COPD. ${ }^{9}$ After discharge, $10 \%$ to $20 \%$ of COPD patients are readmitted within 30 days. ${ }^{10,11}$ Patients who are readmitted following a COPD hospitalization are at greater risk of mortality and have worse outcomes relative to patients who are not readmitted. ${ }^{12}$ A number of interventions have been proposed to reduce readmissions. ${ }^{13}$ However, these interventions have not shown a reduction in readmissions and mortality. ${ }^{13}$ One reason may be the inability to identify patients at the greatest risk of readmission and to target resources to these high-risk patients.

Recently, hospital administrators and the health care community have focused more attention on readmissions among patients with COPD. In an effort to reduce costs and increase quality in health care, the Centers for Medicare and Medicaid Services will penalize hospitals for excess all-cause readmissions within the 30 days following discharge. ${ }^{14}$ Starting in 2015, hospitals with excessive readmissions of patients with COPD face the loss of up to $3 \%$ of total Medicare reimbursement per year. ${ }^{14,15}$ Not surprisingly, this policy change has coincided with an increased interest in estimating the risk of readmissions for COPD-related hospitalizations.

In an effort to expand work beyond single-center studies, researchers have used large, secondary data sources, such as private insurance ${ }^{11}$ and Medicare claims. ${ }^{10}$ However, these data sources have significant limitations depending on the question at hand. Medicare data, for instance, provides little information about the readmission risk factors for patients younger than 65 , and private claims data provides limited information about patients on Medicare, Medicaid or the uninsured. Yet, age and payer status may be important predictors of readmission risk. The purpose of this paper was to examine the risk for readmission within 30 days from time of discharge in patients with COPD using the Healthcare Cost and Utilization Project (HCUP) from the Agency for Healthcare Research and Quality (AHRQ). This statewide dataset allows us to consider all readmissions for COPD regardless of age or payer status.

\section{Methods}

\section{Data Source}

The primary data source for this project was the AHRQ HCUP State Inpatient Database for California for 2005 to 2011. The HCUP State Inpatient Database captures $100 \%$ of all non-federal discharges in a state in a given year. The dataset includes a unique, persistent patient identifier that allows users to track patients across visits. The total dataset included 28,265,070 visits among $17,918,374$ patients over 480 hospitals.

\section{Population}

We identified patients with a hospitalization with a primary diagnosis related to COPD (specifically, an International Classification of Disease, 9 th edition code of 490, 491.0, 491.1, 491.2, 491.20, 491.21, 491.22, $491.8,491.9,492.0,492.8$ or 496$)$, age 40 or older and discharged alive. This case definition is similar to that used by previous studies of COPD using administrative data and the Nationwide Inpatient Sample. ${ }^{11,16}$ We found 292,425 hospitalizations that matched this definition of a COPD hospitalization that also included our covariates of interest.

Of the 292,425 hospitalizations, $74.13 \%$ have a primary diagnoses code of 491.21 , obstructive chronic bronchitis with acute exacerbation. An additional 16.82\% were coded as 491.22, obstructive chronic bronchitis with acute bronchitis. The other codes accounted for the remaining $9.05 \%$ with only 490 , bronchitis not specified as acute or chronic, accounting for more than $3 \%$ of the events. Of these visits, 6112 were visits that included the death of the patient, leaving 286,313. A step-bystep overview of the sample size is reported in Table 1 and summary statistics for the final sample population are included in Table 2. The number of admissions, 


\section{Table 1. Sample Size and Exclusion Rules Applied}

\begin{tabular}{lrr} 
Step & \multicolumn{1}{c}{$\begin{array}{c}\text { Sample } \\
\text { Size }\end{array}$} & $\begin{array}{c}\text { Percent of } \\
\text { Previous } \\
\text { Step }\end{array}$ \\
\hline HCUP SID for CA & $28,265,070$ & - \\
\hline COPD Hospitalizations & 300,084 & $1.06 \%$ \\
\hline Complete Covariates & 297,790 & $99.24 \%$ \\
\hline Age $>\mathbf{4 0}$ & 292,425 & $98.20 \%$ \\
\hline
\end{tabular}

readmission rate by month and mortality rates are included in Figure 1.

\section{Identification of Readmissions}

The interval between 2 stays for a patient was calculated by considering the number of days between the admission date of the current stay and the latest discharge date of the prior stays for that patient. We only considered readmissions occurring between 1 and 30 days after discharge. We did not include sameday readmissions due to transfers and administrative changes, such as moving between units. Using the interval of 1-30 days we can be assured that these reflect true readmissions and not simply patient movement between units or hospitals. Of the 286,313 complete records, 35,030 (12.2\%) were associated with at least one re-hospitalization for any reason within 1-30 days.

\section{Modeling}

To model the dichotomous outcome of readmission within 1 to 30 days, we used a generalized linear model (GLM) based on a Bernoulli outcome with a logit link function. We divided age into 2 groups, under and over 65 , for use as a covariate in the model. Other covariates included gender, presence of do-not-resuscitate orders, primary payer, number of diagnoses, number of procedures, length of stay (log transformed, after adding one), admission month, admission year, admission source (e.g., emergency department, routine, long-term care), discharge destination (e.g., home, transfer, longterm care) and the 29 Elixhauser comorbidities. ${ }^{17} \mathrm{We}$ also included an interaction term between the primary payer and age group, given the differences in expected health status between Medicare patients under the age of 65 and those over 65 .

We only observed post-discharge mortality that occurred during an inpatient stay: if the individual died in the community, long-term care or the emergency department, we did not observe the death in these data. It is possible that this unobserved mortality varied by age, influencing our results. While we do not know if the patient died after discharge, it seems likely that inpatient mortality would be correlated with mortality shortly following discharge. We attempt to capture this relationship by computing age-dependent rates of inpatient mortality. Specifically, we estimated the unadjusted probability of inpatient mortality for each 10-year age group (e.g., 40-49, 50-59, ...) and included these age-specific mortality probabilities as a continuous variable in the model.

Because of the potential for clustering effects within hospital and patient, we used empirical (or robust) standard errors, which are consistent in the presence of such effects.

To further examine the relationship between readmission, payer status and age, using the results from the model, we calculated the predicted readmission risk for each patient and grouped the results by payer status/ age group categories.

\section{Results}

The summary statistics in Table 2 highlight some interesting unadjusted results. The age distribution between the patients who are readmitted and not readmitted is different. Younger patients were more likely to be readmitted than older patients, with a maximal risk of readmission occurring in middle age (40-60 years old). This finding persists after adjustment for severity and inpatient mortality. Full regression results based on the multivariable generalized linear model are shown in Table 3 . Additionally, the plots in Figure 1 indicate that the primary admission rate is seasonal with a winter spike; however, the readmission rate does not have the same pattern but rather peaks during the summer. This differing seasonality is robust to adjustment for severity and other possible confounders. Compared to January, readmission rates are higher from May to November. Specifically, these months have odds of readmission within 30 days that are $8 \%-12 \%$ higher than January.

Notable other highlights from these results include the increased risk of readmission among COPD patients under age 65 (odds ratio [OR] = 1.09, $p=0.00004,95 \%$ confidence interval $[\mathrm{CI}]=[1.04,1.15])$ and the effects of payer status interacted with age group. While there is not a substantial change in readmission risk for the older patients based on payer, for younger patients there 


\section{Table 2. Summary Statistics of COPD Hospitalizations by Readmission Status}

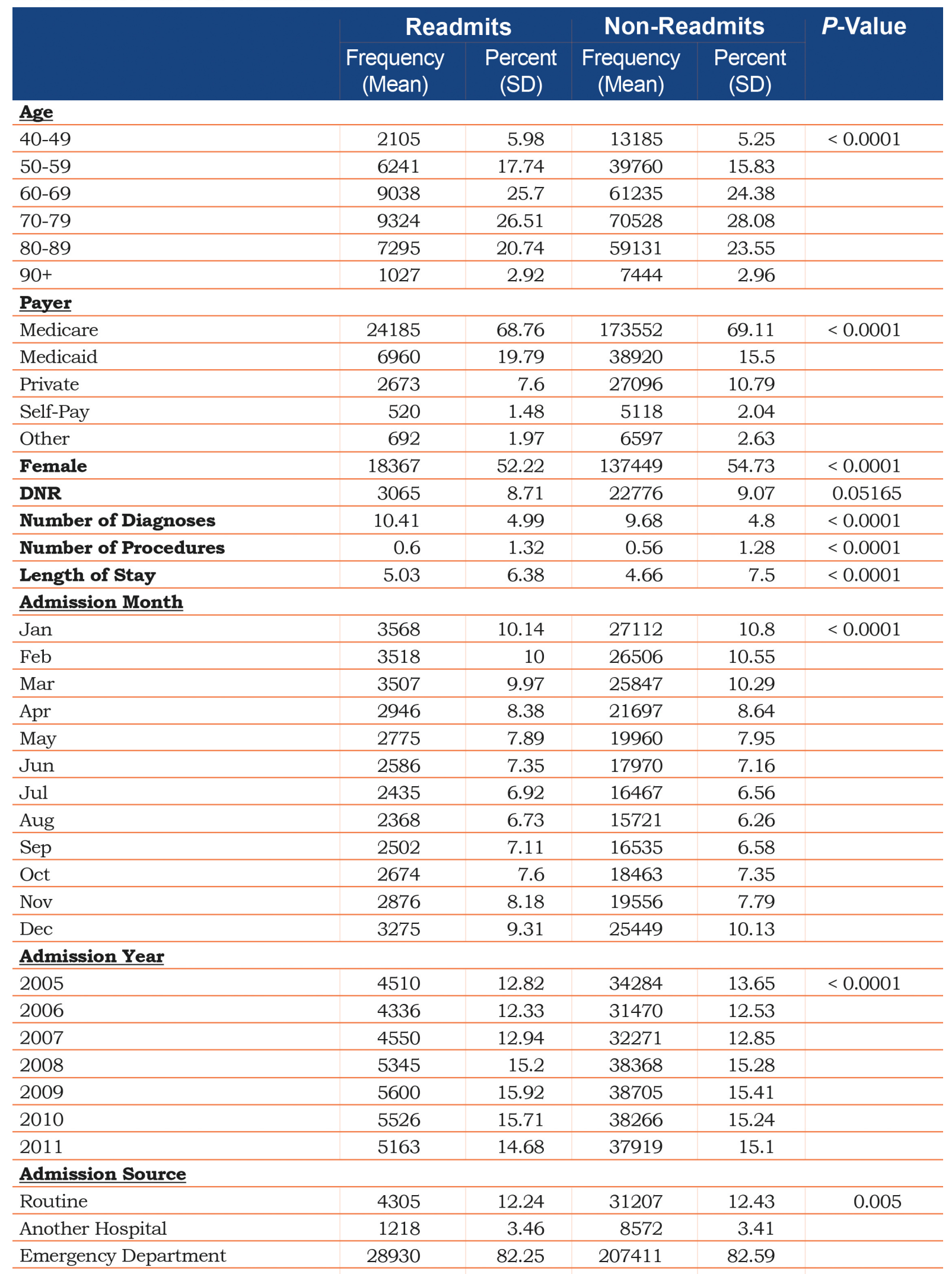




\begin{tabular}{|c|c|c|c|c|c|}
\hline LTC/Other Facility & 572 & 1.63 & 3932 & 1.57 & \\
\hline Court/Law Enforcement & $<11$ & $<0.03$ & 161 & 0.06 & \\
\hline \multicolumn{6}{|l|}{ Discharge Status } \\
\hline Routine & 21910 & 62.29 & 165555 & 65.92 & $<0.0001$ \\
\hline Another Hospital & 204 & 0.58 & 4903 & 1.95 & \\
\hline LTC/Other Facility & 6333 & 18.01 & 42451 & 16.9 & \\
\hline Home Health Care & 5340 & 15.18 & 32760 & 13.04 & \\
\hline AMA & 1182 & 3.36 & 4711 & 1.88 & \\
\hline Unknown but alive & 903 & 2.57 & 903 & 0.36 & \\
\hline Number of Elixhauser Comorbidities & 2.9 & 1.76 & 2.6 & 1.71 & $<0.0001$ \\
\hline \multicolumn{6}{|l|}{ Elixhauser Comorbidities } \\
\hline Aids & 144 & 0.41 & 752 & 0.3 & 0.0018 \\
\hline Alcohol & 2128 & 6.07 & 12392 & 4.93 & $<0.0001$ \\
\hline Deficiency Anemias & 7536 & 21.51 & 43624 & 17.36 & $<0.0001$ \\
\hline RA/Collagen Vascular Diseases & 984 & 2.81 & 6769 & 2.69 & 0.022 \\
\hline Blood Loss & 229 & 0.65 & 1264 & 0.5 & 0.0008 \\
\hline Congestive Heart Failure & 10994 & 31.38 & 63919 & 25.44 & $<0.0001$ \\
\hline Chronic Lung Disease & 66 & 0.19 & 505 & 0.2 & 0.613 \\
\hline Coagulation Disorder & 909 & 2.59 & 5411 & 2.15 & $<0.0001$ \\
\hline Depression & 4551 & 12.99 & 30444 & 12.12 & $<0.0001$ \\
\hline Diabetes & 8930 & 25.49 & 57937 & 23.06 & $<0.0001$ \\
\hline Diabetes with Complications & 1970 & 5.62 & 11582 & 4.61 & $<0.0001$ \\
\hline Drug Abuse & 2185 & 6.24 & 11162 & 4.44 & $<0.0001$ \\
\hline Hypertension with Complications & 21934 & 62.61 & 156425 & 62.25 & 0.1868 \\
\hline Hypothyroidism & 4355 & 12.43 & 31132 & 12.39 & 0.8193 \\
\hline Liver Disease & 1430 & 4.08 & 7409 & 2.95 & $<0.0001$ \\
\hline Lymphoma & 220 & 0.63 & 1416 & 0.56 & 0.1495 \\
\hline Fluid and Electrolyte Disorder & 8513 & 24.3 & 57606 & 22.92 & $<0.0001$ \\
\hline Metastatic Cancer & 631 & 1.8 & 3059 & 1.22 & $<0.0001$ \\
\hline Neurological Disorder & 2849 & 8.13 & 18810 & 7.49 & $<0.0001$ \\
\hline Obesity & 4677 & 13.35 & 31549 & 12.56 & $<0.0001$ \\
\hline Paralysis & 636 & 1.82 & 4409 & 1.75 & 0.4222 \\
\hline Peripheral Vasuclar Disorder & 2619 & 7.48 & 18343 & 7.3 & 0.2382 \\
\hline Psychoses & 3512 & 10.03 & 19060 & 7.59 & $<0.0001$ \\
\hline Pulmonary/Circulatory Disorder & 156 & 0.45 & 1014 & 0.4 & 0.2682 \\
\hline Renal Failure & 4420 & 12.62 & 26299 & 10.47 & $<0.0001$ \\
\hline Ulcer & 22 & 3.18 & 6499 & 2.59 & $<0.0001$ \\
\hline Solid Tumor & 1114 & 0.06 & 166 & 0.07 & 0.8202 \\
\hline Valvular Tumor & 22 & 5.91 & 13979 & 5.56 & 0.0098 \\
\hline Weight Loss & 2070 & 4.82 & 10017 & 3.99 & $<0.0001$ \\
\hline
\end{tabular}

Numbers are counts followed by percentages, with the exception of the number of diagnoses, the number of procedures, the length of stay and the Elixhauser comorbidity count, which are means followed by standard deviations.

$P$-values correspond to t-tests for continuous variables and chi-squared test for categorical variables.

is a significant and sizable difference in readmission risk based on payer status. Thus, a patient under 65 with private insurance has 0.67 (95\% CI: 0.61-0.73) times the odds of being readmitted relative to a patient under 65 on Medicare. Patients under 65 on Medicaid have a 1.19 (95\% CI: 1.10-1.29) fold increase in their odds of readmission relative to patients under 65 on Medicare.
In addition, the likelihood of being readmitted for any admission and for COPD specifically are similar with a rapid decrease in risk over the first several days followed by a more gradual decrease, as illustrated in Figure 2. Note, however, that for any given number of days after discharge, COPD admissions are more likely to result in a readmission than a typical admission. 


\section{Figure 1. Admission Frequency, Readmission Rate and Inpatient Mortality Rate by Month from 01/1998 to $11 / 2011$}
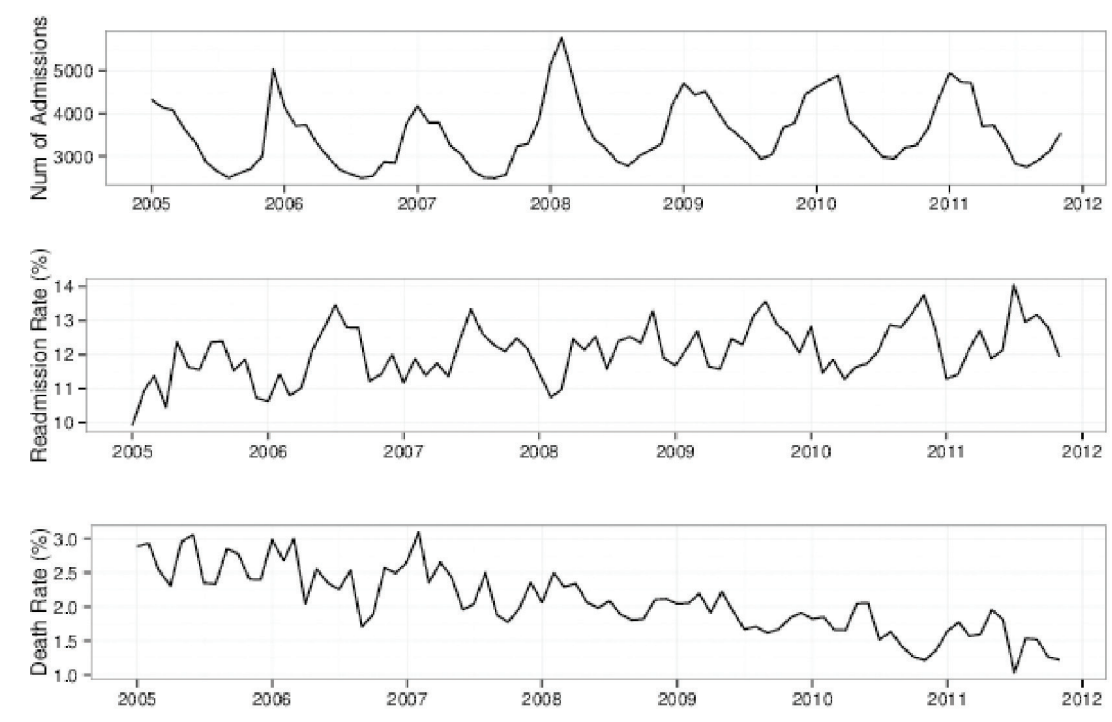

December 2011 was not included due to censoring of admissions that are not discharged in 2011. Admission volume spikes during the winter and is correlated with the influenza season. However, these winter admissions are not more likely to return. Summer readmissions are the most likely to return within 30 days. Inpatient mortality is not strongly seasonal and decreasing over the interval .

be seasonal.

\section{Discussion}

Our results show that younger patients with COPD are more likely than older patients to be readmitted, even after controlling for observable comorbidities. Payer status also affects the chances of patients being readmitted: younger people with private insurance are the least likely to be readmitted. Interestingly, these observations would have been more difficult to identify in datasets defined by payer type. For example, with Medicare, most patients are over the age of 65 , and with private insurance plans, most patients are under 65, and many are employed. Thus, using convenient sources of administrative data linked to payer status may obscure important

For ease of interpretation, the mean computed readmission probability grouped by age and payer are reported in Table 4. Readmission rates for younger patients differ by insurance type. Younger patients on Medicaid have a nearly 6 percentage point increase in risk of 30-day readmission relative to older patients on Medicaid, and an 8 percentage point increase relative to young patients on private insurance. Additionally, these patients have readmission probabilities 1.5 percentage points higher than young patients on Medicare. On the other hand, readmission rates for older patients do not differ by insurance type. The range among the patients older than 65 between payers is under 2 percentage points (9.89\% for self-pay to $11.80 \%$ for Medicare). In addition, the risk of readmission is elevated for those discharged to home health care or who leave against medical advice, as compared to those discharged to home.

Figure 1 highlights changes in volume, readmission and mortality in the COPD population over time. No significant changes occur in the number of COPD admissions in our data but there is a sizable reduction in the inpatient mortality of these patients and a potential upward trend in the fraction of patients being readmitted. Finally, the inpatient mortality rate changes considerably over the study interval from 3\% to $1.25 \%$ as shown in Figure 1. The death rate does not appear to risk factors for 30-day readmission. Many of the other risk factors we identified were related to either aspects of socioeconomic status, (e.g., race) or preexisting comorbidities. In general, most of these risk factors are related to the patient rather than the quality of care received. This may help explain why many of the interventions designed to prevent readmissions have not been successful.

Our finding that younger patients are more likely to be readmitted may seem counterintuitive. However, summary statistics in prior analyses have also shown an increased risk among younger patients. ${ }^{16,18} \mathrm{We}$ speculate that this finding is due, in part, to a survivor effect: for younger patients to be admitted for COPD, their disease must be very severe. Patients who have survived to an older age may have milder forms of COPD or a much slower rate of disease progression. In addition, interactions between age and payer status also help explain this observation. The least likely to be readmitted are younger patients with private insurance. In fact, younger patients on private insurance have a $38 \%$ reduction in the odds of being readmitted relative to younger patients on Medicare. As private insurance is nearly synonymous with employer-sponsored insurance, patients on private insurance are likely either well enough to work themselves or have a partner well enough to be employed. Their disease state and other 


\section{Table 3. Regression Results with Conference Intervals for 30-Day All-Cause Readmission Following COPD Hospitalization}

\begin{tabular}{|c|c|c|c|c|}
\hline \multirow{2}{*}{$\begin{array}{l}\text { Variable } \\
\text { Age < } 65\end{array}$} & \multicolumn{3}{|c|}{$\begin{array}{l}\text { Odds } \quad 95 \% \text { Cl } \\
\text { Ratio Lower Upper }\end{array}$} & $P$ \\
\hline & 1.09 & 1.04 & 1.15 & 0.0005 \\
\hline Female & 0.91 & 0.89 & 0.93 & $<0.0001$ \\
\hline Number of Diagnoses & 1.01 & 1.01 & 1.02 & $<0.0001$ \\
\hline Number of Procedures & 0.98 & 0.97 & 0.99 & 0.0002 \\
\hline \multicolumn{5}{|l|}{ Admission Source (Routine) } \\
\hline Another Hospital & 1.03 & 0.96 & 1.10 & 0.4428 \\
\hline Court/Jail/Law Enforcement & 0.29 & 0.12 & 0.72 & 0.0075 \\
\hline Emergency Room & 1.03 & 0.99 & 1.06 & 0.1192 \\
\hline Other/Long Term Care & 0.95 & 0.87 & 1.05 & 0.3320 \\
\hline \multicolumn{5}{|l|}{ Payer (Medicare) } \\
\hline Medicaid & 0.96 & 0.89 & 1.03 & 0.2236 \\
\hline Private & 0.90 & 0.84 & 0.95 & 0.0006 \\
\hline Self-Pay & 0.89 & 0.69 & 1.16 & 0.3925 \\
\hline Other & 0.87 & 0.71 & 1.07 & 0.1865 \\
\hline \multicolumn{5}{|l|}{ Under 65 Interaction With... } \\
\hline Medicaid & 1.19 & 1.10 & 1.29 & $<0.0001$ \\
\hline Private & 0.67 & 0.61 & 0.73 & $<0.0001$ \\
\hline Self-Pay & 0.73 & 0.55 & 0.96 & 0.0252 \\
\hline Other & 0.77 & 0.62 & 0.97 & 0.0244 \\
\hline \multicolumn{5}{|l|}{ Discharge (Home) } \\
\hline Home Health Care & 1.16 & 1.12 & 1.20 & $<0.0001$ \\
\hline Long-Term Care/Skilled Care & 1.01 & 0.98 & 1.04 & 0.6001 \\
\hline Transfer to Another Hospital & 0.29 & 0.25 & 0.33 & $<0.0001$ \\
\hline AMA & 1.77 & 1.66 & 1.90 & $<0.0001$ \\
\hline Unknown & 0.44 & 0.34 & 0.58 & $<0.0001$ \\
\hline DNR & 0.97 & 0.93 & 1.01 & 0.1331 \\
\hline $\log (\operatorname{LOS}+1)$ & 1.16 & 1.13 & 1.18 & $<0.0001$ \\
\hline \multicolumn{5}{|l|}{ Month (Jan) } \\
\hline Feb & 1.02 & 0.97 & 1.07 & 0.5144 \\
\hline Mar & 1.03 & 0.98 & 1.08 & 0.2725 \\
\hline Apr & 1.02 & 0.97 & 1.08 & 0.3580 \\
\hline May & 1.05 & 0.99 & 1.10 & 0.0926 \\
\hline June & 1.08 & 1.02 & 1.14 & 0.0067 \\
\hline July & 1.10 & 1.04 & 1.16 & 0.0010 \\
\hline Aug & 1.11 & 1.05 & 1.18 & 0.0002 \\
\hline Sep & 1.12 & 1.06 & 1.18 & 0.0001 \\
\hline Oct & 1.07 & 1.01 & 1.13 & 0.0131 \\
\hline Nov & 1.09 & 1.03 & 1.15 & 0.0017 \\
\hline Dec & 0.97 & 0.92 & 1.02 & 0.2686 \\
\hline Year & 0.99 & 0.98 & 1.00 & 0.0010 \\
\hline \multicolumn{5}{|l|}{ Race (White) } \\
\hline Black & 1.20 & 1.16 & 1.25 & $<0.0001$ \\
\hline
\end{tabular}

unobserved health effects are not significant enough to prevent them from working, or if the disease is advanced enough, patients with private insurance likely have a well partner who may be able to provide some in-home care. In contrast, patients under the age of 65 on Medicare are likely on disability due to their disease state or some other health-related factors unobservable in administrative data. Even after adjustment for the Elixhauser comorbidities ${ }^{17}$ and a number of other markers for severity, patients on Medicare or Medicaid under the age of 65 are much more likely to return to the hospital within 30 days. We think that the Medicare/Medicaid effect in middle-aged patients is due to the initial severity required to be eligible for Medicaid or Medicare in middle age. These patients differ greatly from middle-aged patients with private insurance. We do not observe a similar payer effect among the elderly. Because all elderly patients are eligible for Medicare, the elderly may not vary in health status among payers as much as younger patients do.

The differences we observe in readmission with regard to insurance indicate that merely providing patients access to health care coverage may not be enough to prevent readmissions. A patient on Medicare or private insurance, for example, should have similar levels of access to care. However, younger patients without insurance are less likely to be readmitted in 30 days than those with Medicare or Medicaid. This implies that patient health is probably a more important factor than insurance coverage in predicting readmission.

Admissions for COPD are seasonal, and are strongly associated with the influenza season in the prior literature. ${ }^{19-22}$ However, we did not observe a similar pattern of seasonality in 30-day readmissions risk. Rather, readmission risk was elevated during the summer and early-fall and at a minimum during the winter months. Admissions not driven by respiratory viruses may be fundamentally different from exacerbations that are driven by environmental factors or the underlying disease itself. Alternatively, some of these winter admissions for COPD may be misclassified and may be part of the viral process of influenza-like illness. In any event, strategies to prevent readmission may need to be incorporated throughout the year, not only during the influenza season and these approaches may need to vary across the year to reduce readmission risk. 


\begin{tabular}{|c|c|c|c|c|}
\hline Hispanic & 0.92 & 0.89 & 0.96 & $<0.0001$ \\
\hline Asian/Pacific Islander & 0.95 & 0.90 & 1.00 & 0.0644 \\
\hline Native American & 1.75 & 1.24 & 2.46 & 0.0015 \\
\hline Other & 0.73 & 0.68 & 0.78 & $<0.0001$ \\
\hline \multicolumn{5}{|l|}{ Elixhauser Comorbidities } \\
\hline Aids & 1.01 & 0.84 & 1.21 & 0.9513 \\
\hline Alcohol & 1.08 & 1.03 & 1.14 & 0.0019 \\
\hline Deficiency Anemias & 1.17 & 1.13 & 1.20 & $<0.0001$ \\
\hline RA/Collagen Vascular Diseases & 1.02 & 0.95 & 1.09 & 0.6369 \\
\hline Blood Loss & 1.18 & 1.02 & 1.36 & 0.0271 \\
\hline Congestive Heart Failure & 1.25 & 1.22 & 1.29 & $<0.0001$ \\
\hline Chronic Lung Disease & 0.86 & 0.66 & 1.13 & 0.2828 \\
\hline Coagulation Disorder & 1.01 & 0.94 & 1.08 & 0.8603 \\
\hline Depression & 1.04 & 1.00 & 1.07 & 0.0402 \\
\hline Diabetes & 1.09 & 1.06 & 1.12 & $<0.0001$ \\
\hline Diabetes with Complications & 1.10 & 1.04 & 1.16 & 0.0004 \\
\hline Drug Abuse & 1.11 & 1.05 & 1.17 & 0.0001 \\
\hline Hypertension with Complications & 0.96 & 0.93 & 0.98 & 0.0007 \\
\hline Hypothyroidism & 1.01 & 0.97 & 1.04 & 0.6633 \\
\hline Liver Disease & 1.13 & 1.06 & 1.20 & 0.0001 \\
\hline Lymphoma & 1.08 & 0.94 & 1.25 & 0.2774 \\
\hline Fluid and Electrolyte Disorder & 0.98 & 0.96 & 1.01 & 0.2150 \\
\hline Metastatic Cancer & 1.48 & 1.35 & 1.61 & $<0.0001$ \\
\hline Neurological Disorder & 1.01 & 0.97 & 1.06 & 0.5041 \\
\hline Obesity & 0.90 & 0.87 & 0.93 & $<0.0001$ \\
\hline Paralysis & 0.95 & 0.87 & 1.03 & 0.2262 \\
\hline Peripheral Vasuclar Disorder & 0.95 & 0.91 & 0.99 & 0.0217 \\
\hline Psychoses & 1.19 & 1.14 & 1.24 & $<0.0001$ \\
\hline Pulmonary/Circulatory Disorder & 0.87 & 0.73 & 1.04 & 0.1305 \\
\hline Renal Failure & 1.09 & 1.05 & 1.13 & $<0.0001$ \\
\hline Solid Tumor & 1.24 & 1.16 & 1.33 & $<0.0001$ \\
\hline Valvular Tumor & 0.97 & 0.92 & 1.02 & 0.1840 \\
\hline Ulcer & 0.88 & 0.56 & 1.38 & 0.5833 \\
\hline Weight Loss & 1.08 & 1.03 & 1.15 & 0.0042 \\
\hline Inpatient Mortality Rate (Percent) & 0.92 & 0.90 & 0.94 & $<0.0001$ \\
\hline
\end{tabular}

reduced by discharging them to skilled care instead of home health. Further investigation of the tradeoff between skilled care and home health care is warranted.

An additional area for future investigations is to explore the relationship between length of stay and readmission for high-risk patients. For example, it may be that some of the patients who went to home health services would be less likely to be readmitted if they had stayed longer during their first hospital admission. The same is true for those who leave against medical advice. Such patients may constitute the highest yield populations for decreasing readmissions. Increasing the lengths of stay in the original hospitalization may lead to fewer readmissions and better outcomes. Finally, some of the comorbidities that we identify, including congestive heart failure and diabetes, can be improved with management. Thus, comorbidities that can be managed and improved during hospitalization and following discharge should be targeted for interventions designed to reduce readmission. For example, clinical trials can be directed towards this high risk population with the intention to reduce COPD readmission. Also, extra efforts could be made to provide pulmonary rehabilitation, an effective treatment for improving symptoms related to COPD. ${ }^{23}$

Finally, we observe a drastic reduction in inpatient mortality over the study interval, a change not observed among the entire patient population as a whole (data not shown). The cause of this reduction is not immediately clear. It may be due to the shifting of mortality to the outpatient setting (e.g., hospice and similar end-oflife care) or improvement in treatment quality and effectiveness. Future work is needed to understand Labels provided in parentheses are reference levels for odds ratios.

Many efforts to predict hospital readmission have been challenging. ${ }^{13}$ and although many of the risk factors are difficult to control, our results suggest that higherrisk patients are identified at the time of discharge. For example, patients that are assigned home health care or that leave against medical advice are much more likely to be readmitted compared to patients discharged to home or a skilled nursing care. It seems reasonable that patients discharged with home health care are more ill or have less social support than patients discharged to home without home health care while being somewhat less ill than those sent to skilled care after discharge. For some of these patients, readmission risk may be the causes of this reduction in inpatient mortality among COPD patients.

Our work is subject to several limitations. First, the data we used were administrative in nature and include no information about the following variables: smoking status, specific laboratory results, imaging, pulmonary function testing, COPD staging or medication use-variables that are important to consider when studying COPD. Second, our results may be driven by censoring due to mortality. Mortality varies by age group and generally increases with age. We included inpatient mortality in the model and the effect was strongly 
Figure 2. Percent of All Patients Readmitted by
Days from Discharge and Admission Type

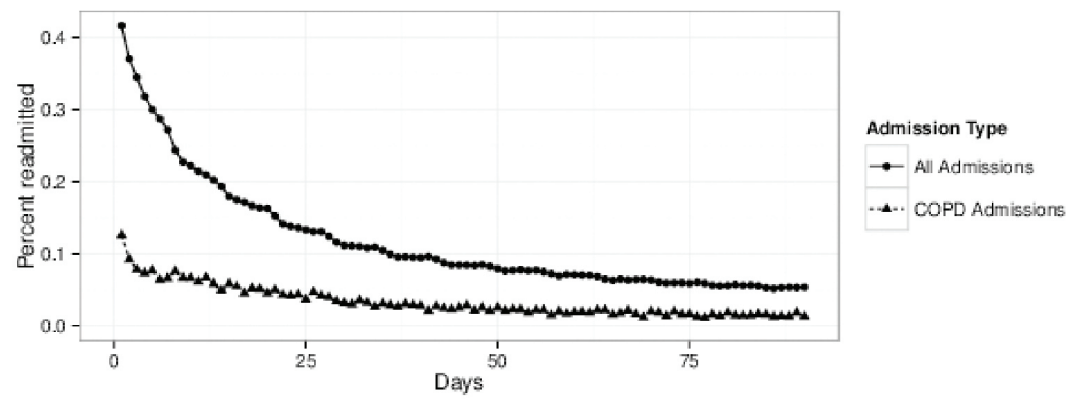

Patients admitted for COPD are more likely to return than a randomly selected patient. The shape of the 2 curves are similar otherwise.

Table 4. Mean Predicted Probability of Readmission by Payer and Age Group

\begin{tabular}{lcc} 
& Age $<65$ & Age $\geq 65$ \\
Medicare & $14.77 \%$ & $11.80 \%$ \\
\hline Medicaid & $16.27 \%$ & $10.89 \%$ \\
\hline Private & $8.25 \%$ & $10.12 \%$ \\
\hline Self-Pay & $9.13 \%$ & $9.89 \%$ \\
\hline Other & $9.39 \%$ & $10.11 \%$ \\
\hline
\end{tabular}

significant. Each percentage point increase in the mortality rate is associated with a nearly $10 \%$ decrease in the odds of readmission, even after requiring the patient to be discharged alive. This effect is presumably due to unobserved mortality: people who die cannot be readmitted. By including age-specific inpatient-mortality rates, we can partially control for the differences in post-discharge mortality rates. Furthermore, it is likely that mortality risk for an AECOPD follows a skewed distribution with the risk of death decreasing as the amount of time since the onset of the exacerbation increases. Patients who survive long enough and are stable enough to be discharged may be much less likely to die in the next 30 days than in the days spent as an inpatient. This would tend to dampen the effect of censoring due to unobserved postdischarge mortality.

Despite these limitations, our results demonstrate how many of the risk factors for readmission are clearly related to patients themselves, rather than the quality or process of care that a patient receives during a hospital admission for COPD. This observation may help explain why prior programs to reduce readmissions have not been successful. In addition, our results can help design future interventions. Such interventions should target younger patients with Medicare or Medicaid coverage, with multiple comorbidities, and patients who leave the hospital with either home health or against medical advice. Because many of these patient-based features may not be easy to control, and since the Medicare payment implications may have adverse effects on hospitals, future work should focus on characterizing the degree to which readmission risk is associated with patient-level factors as opposed to quality of care.

\section{Declaration of Interest}

All authors report no conflicts of interest. 


\section{References}

1. Beran D, Zar HJ, Perrin C, Menezes AM, Burney P. Burden of asthma and chronic obstructive pulmonary disease and access to essential medicines in low-income and middle-income countries. Lancet Respir Med. 2015;3(2):159-170. doi: http://dx.doi.org/10.1016/S2213-2600(15)00004-1

2. Vestbo J, Hurd SS, Agusti AG, et al. Global strategy for the diagnosis, management, and prevention of chronic obstructive pulmonary disease: GOLD executive summary. Am J Respir Crit Care Med. 2013;187(4):347-365.

doi: http://dx.doi.org/10.1164/rccm.201204-0596PP

3. Carolan BJ, Hughes G, Morrow J, et al. The association of plasma biomarkers with computed tomography-assessed emphysema phenotypes. Respir Res. 2014;15:127. doi: http://dx.doi.org/10.1186/s12931-014-0127-9

4. Castaldi PJ, Dy J, Ross J, et al. Cluster analysis in the COPDGene study identifies subtypes of smokers with distinct patterns of airway disease and emphysema. Thorax. 2014;69(5):415-422. doi: http://dx.doi.org/10.1136/thoraxjnl-2013-203601

5. Hansel NN, Washko GR, Foreman MG, et al. Racial differences in CT phenotypes in COPD. COPD. 2013;10(1):20-27. doi: http://dx.doi.org/10.3109/15412555.2012.727921

6. Wan ES, Silverman EK. Genetics of COPD and emphysema. Chest. 2009;136(3):859-866. doi: http://dx.doi.org/10.1378/chest.09-0555

7. Han MK, Kazerooni EA, Lynch DA, et al. Chronic obstructive pulmonary disease exacerbations in the COPDGene study: associated radiologic phenotypes. Radiology. 2011;261(1):274282. doi: http://dx.doi.org/10.1148/radiol.11110173

8. Wedzicha JA, Singh R, Mackay AJ. Acute COPD exacerbations. Clin Chest Med. 2014;35(1):157-163.

doi: http://dx.doi.org/10.1016/j.ccm.2013.11.001

9. Marchetti N,Criner GJ,AlbertRK. Preventing acute exacerbations and hospital admissions in COPD. Chest. 2013;143(5):1444-1454. doi: http://dx.doi.org/10.1378/chest.12-1801

10. Shah T, Churpek MM, Perraillon MC, Konetzka RT. Understanding why COPD patients get readmitted: A large national study to delineate the Medicare population for the readmissions penalty expansion. Chest. 2015;147(5): 1219-1226. doi: http://dx.doi.org/101378/chest.14218

11. Sharif R, Parekh TM, Pierson KS, Kuo Y-F, Sharma G. Predictors of early readmission among patients 40 to 64 years of age hospitalized for chronic obstructive pulmonary disease. Ann Am Thorac Soc. 2014;11(5):685-694.

doi: http://dx.doi.org/10.1513/AnnalsATS.201310-358OC
12. Almagro P, Calbo E, de Echagüen AO, et al. Mortality after hospitalization for COPD. Chest. 2002;121(5):1441-1448. doi: http://dx.doi.org/10.1378/chest.121.5.1441

13. Prieto-Centurion V, Markos MA, Ramey NI, et al. Interventions to reduce rehospitalizations after chronic obstructive pulmonary disease exacerbations. A systematic review. Ann Am Thorac Soc. 2014;11(3):417-424.

doi: http://dx.doi.org/10.1513/AnnalsATS.201308-254OC

14. Joynt KE, Jha AK. A path forward on Medicare readmissions. New Eng J Med. 2013;368(13):1175-1177. doi: http://dx.doi.org/10.1056/NEJMp1300122

15. Feemster LC, Au DH. Penalizing hospitals for chronic obstructive pulmonary disease readmissions. Am J Respir Crit Care Med. 2014; 189(6):634-639.

doi: http://dx.doi.org/10.1164/rccm.201308-1541PP

16. Wier L, Elixhauser A, Pfuntner A, Au D. Overview of hospitalizations among patients with COPD, 2008--Statistical Brief\# 106. In: Healthcare Cost and Utilization Project Statistical Briefs. Rockville, MD: Agency for Healthcare Research and Quality;2011. http://www.ncbi.nlm.nih.gov/books/NBK53969/ Accessed May 2016.

17. Elixhauser A, Steiner C, Harris DR, Coffey RM. Comorbidity measures for use with administrative data. Med Care. 1998;36(1):8-27.

doi: http://dx.doi.org/10.1097/00005650-199801000-00004

18. Groenewegen KH, Schols AM, Wouters EF. Mortality and mortality-related factors after hospitalization for acute exacerbation of COPD. Chest. 2003;124(2):459-467. doi: http://dx.doi.org/10.1378/chest.124.2.459

19. Gorse GJ, O'Connor TZ, Young SL, et al. Impact of a winter respiratory virus season on patients with COPD and association with influenza vaccination. Chest. 2006;130(4):1109-1116. doi: http://dx.doi.org/10.1378/chest.130.4.1109

20. Seemungal T, Harper-Owen R, Bhowmik A, et al. Respiratory viruses, symptoms, and inflammatory markers in acute exacerbations and stable chronic obstructive pulmonary disease. Am J Respir Crit Care Med. 2001;164(9):1618-1623. doi: http://dx.doi.org/10.1164/ajrccm.164.9.2105011

21. Glezen WP, Greenberg SB, Atmar RL, Piedra PA, Couch RB. Impact of respiratory virus infections on persons with chronic underlying conditions. JAMA. 2000;283(4):499-505. doi: http://dx.doi.org/10.1001/jama.283.4.499

22. Rohde G, Wiethege A, Borg I, et al. Respiratory viruses in exacerbations of chronic obstructive pulmonary disease requiring hospitalisation: a case-control study. Thorax. 2003;58(1):37-42. doi: http://dx.doi.org/10.1136/thorax.58.1.37

23. McCarthy B, Casey D, Devane D, Murphy K, Murphy E, Lacasse Y. Pulmonary rehabilitation for chronic obstructive pulmonary disease. Cochrane Database Syst Rev. 2015;2:Cd003793. doi: http://dx.doi.org/10.1002/14651858.cd003793.pub3 